\title{
Mirroring the Self: Testing Neurophysiological Correlates of Disturbed Self-Experience in Schizophrenia Spectrum
}

Article in Psychopathology · April 2015

DOI: $10.1159 / 000380884$

\section{CITATIONS}

5

11 authors, including:

Vittorio Gallese

Università degli studi di Parma

304 PUBLICATIONS 39,528 CITATIONS

SEE PROFILE

\section{Maria Alessandra Umiltà}

Università degli studi di Parma

64 PUBLICATIONS 3,668 CITATIONS

SEE PROFILE

\section{Andrea Raballo}

University of Oslo

92 PUBLICATIONS 1,056 CITATIONS

SEE PROFILE

\section{Mario Amore}

Università degli Studi di Genova

199 PUBLICATIONS 2,161 CITATIONS

SEE PROFILE

Some of the authors of this publication are also working on these related projects: 


\section{Mirroring the Self: Testing Neurophysiological Correlates of Disturbed Self-Experience in Schizophrenia Spectrum}

\author{
Mariateresa Sestito ${ }^{a, b}$ Andrea Raballo ${ }^{c, d}$ Maria Alessandra Umiltàe \\ Emanuela Leuci $^{f}$ Matteo Tonna $^{f}$ Renata Fortunati ${ }^{f}$ Giancarlo De Paola ${ }^{f}$ \\ Mario Amoreg Carlo Maggini ${ }^{f}$ Vittorio Gallese ${ }^{a}$
}

a Department of Neuroscience, Unit of Physiology, University of Parma, Parma, Italy; ${ }^{\text {b } C o g n i t i v e ~ N e u r o s c i e n c e ~ C e n t e r, ~}$ Unité mixte de recherche 5229, Centre National pour la Recherche Scientifique (CNRS), Bron, France; ${ }^{\mathrm{C}}$ Department of Mental Health, AUSL of Reggio Emilia, Reggio Emilia, Italy; ${ }^{d}$ Psychiatric Center Hvidovre, Copenhagen, Denmark; e Department of Pharmacy, University of Parma, Parma, Italy; ' Department of Neuroscience, Psychiatric Division, University of Parma, Italy; ${ }^{9}$ Department of Neuroscience, Psychiatric Division, University of Genova, Genova, Italy

\begin{tabular}{l} 
C) Free Author \\
COPY - for per- \\
sonal use only \\
ANY DISTRIBUTION OF THIS \\
ARTICIE WITHOUT WRITTEN \\
CONSENT FROM S. KARGER \\
AG, BASEL ISA VIOLATION \\
OF THE COPYRIGHT. \\
Written permission to distrib- \\
ute the PDF will be granted \\
against payment of a per- \\
mission fee, which is based \\
on the number of accesses \\
required. Please contact \\
permission@karger.com \\
\hline
\end{tabular}

\section{Key Words}

Electrophysiology · Emotions · Facial mimicry · Multisensory integration $\cdot$ Schizophrenia $\cdot$ Self-disorders

\begin{abstract}
Background: Self-disorders (SDs) have been described as a core schizophrenia spectrum vulnerability phenotype, both in classic and contemporary psychopathological literature. However, such a core phenotype has not yet been investigated adopting a trans-domain approach that combines the phenomenological and the neurophysiological levels of analysis. The aim of this study is to investigate the relation between SDs and subtle, schizophrenia-specific impairments of emotional resonance that are supposed to reflect abnormalities in the mirror neurons mechanism. Specifically, we tested whether electromyographic response to emotional stimuli (i.e. a proxy for subtle changes in facial mimicry and related motor resonance mechanisms) would predict the occurrence of anomalous subjective experiences (i.e. SDs). Sampling and Methods: Eighteen schizophrenia spectrum (SzSp) patients underwent a comprehensive psychopathological examination and were contextually tested with a multimodal paradigm, recording facial electromyographic
\end{abstract}

activity of muscles in response to positive and negative emotional stimuli. Experiential anomalies were explored with the Bonn Scale for the Assessment of Basic Symptoms (BSABS) and then condensed into rational subscales mapping SzSp anomalous self-experiences. Results: SzSp patients showed an imbalance in emotional motor resonance with a selective bias toward negative stimuli, as well as a multisensory integration impairment. Multiple regression analysis showed that electromyographic facial reactions in response to negative stimuli presented in auditory modality specifically and strongly correlated with SD subscore. Conclusions: The study confirms the potential of SDs as target phenotype for neurobiological research and encourages research into disturbed motor/emotional resonance as possible body-level correlate of disturbed subjective experiences in SzSp.

(c) 2015 S. Karger AG, Basel

\section{Introduction}

Contemporary phenomenologically inspired empirical research indicates that disturbance of the basic sense of self may be a core phenotypic marker of schizophrenia spectrum (SzSp) disorders [1-7]. Indeed in SzSp disor-

\section{KARGER 125}

(c) 2015 S. Karger AG, Base

$0254-4962 / 15 / 0000-0000 \$ 39.50 / 0$

E-Mail karger@karger.com

www.karger.com/psp
Mariateresa Sestito, $\mathrm{PhD}$

Unit of Physiology, Department of Neuroscience, University of Parma

Via Volturno 39

IT-43100 Parma (Italy)

E-Mail mariateresa.sestito@ nemo.unipr.it 
ders, the basic selfhood seems to be subtly perturbed, unstable and oscillating, resulting in often alarming and alienating experiences that frequently date back to childhood and/or early adolescence. The psychopathological notion of self-disorder (SD) specifically refers to such a disturbed sense of the basic self, that entails a variety of anomalous subjective experiences mostly affecting the sense of being a self-present, embodied subject immersed in the world [8]. The 'basic' or 'core' self is indeed a prereflective, tacit level of selfhood. The basic sense of self signifies that we each live our conscious life as a self-present, single, temporally persistent, bodily, and demarcated subject of experience and action. It refers to the implicit first-person quality of consciousness, i.e. the implicit awareness that all experience articulates itself in first-person perspective as 'my' experience, constituting the foundational level of selfhood on which other levels of selfhood are built. From a phenomenological perspective, disturbances of the basic sense of self entail various forms of depersonalization, loss of common sense, fading grip on perceptual and situational meanings, distortions of the stream of consciousness, as well as disordered sense of identity and embodiment. Although different in their thematic content, all these anomalous subjective experiences are characterized by subtle, nonpsychotic qualitative changes in the structure of subjectivity $[9,10]$ implying a fundamental shift in the sense of being a self-coincident subject, endowed with a stable first-person perspective and vitally engaged in the world.

Clinically, SDs encompass a broad range of phenomena that can be reliably explored through systematic checklists such as the Bonn Scale for the Assessment of Basic Symptoms (BSABS) as well as other recent instruments developed from the BSABS, like the Schizophrenia Proneness Instrument (SPI-A, SPI-CY) and the ad hoc constructed Examination of Anomalous Self-Experience (EASE) [11-13].

These not yet psychotic anomalies of subjective experiences antedate the onset of flamboyant psychosis $[8,14-$ 18 ] and occur in various schizophrenia-related conditions (i.e. full-blown schizophrenia, prodromal/at risk mental states, schizotypy) including unaffected schizotaxic subjects and genetically high-risk individuals $[3,5,7,14,19]$. In a recent study [20], the specificity of SDs for the SzSp disorders has been demonstrated in a diagnostically heterogeneous sample, where SDs aggregated with similar levels in schizophrenia and schizotypy. Hence, besides their clinical value for the purposes of early differential diagnosis, SDs constitute a valuable trait phenotype for in- dexing genetic liability to SzSp disorders [21] (see [22] for a review).

Up to now, however, little is known about the electrophysiological bodily correlates of SDs $[23,24]$. This is particularly important given the marked gap between patients' complaints (that generally entail disturbing experiences) and the most prominent schizophrenia etiopathogenetic theories and therapeutic practices (which are by large postulated at a brain level of description and analysis). Until now, nobody has ever found a direct association between phenomenological experience (i.e. the experiential level) as measured by systematic checklists and electrophysiological evidence (i.e. the body level) in schizophrenia disorder. Therefore, the current study was designed to focus on the convergence between contemporary emotion neuroscience and phenomenological investigations of SzSp vulnerability [25].

Recent findings strongly support an impairment of emotional resonance in patients with schizophrenia, likely rooting these deficits to abnormalities in the mirror neurons mechanism [26-30]. Particularly, the disruption in patients with schizophrenia of the low-level mechanism of facial mimicry $[25,31,32]$, which normally occurs when someone perceives the facial expression of someone else's emotion, compromises patients' emotional empathy and understanding [33-36].

A phenomenological account of the implicit functioning of the body in everyday perception and action turns the traditional notion of the physical body into a living medium of the individual's relation to the world $[37,38]$. According to this view, subtle distortions of these embodied mediating processes would be a crucial feature of schizophrenia, which - indeed - has been phenomenologically reconceptualized by Fuchs [39] as a disorder of embodied intersubjectivity [40]. Thus, the aim of this study is to investigate whether a direct connection between congruent motor facial mimicry in response to emotional stimuli and anomalous subjective experiences (i.e. SDs) exists in SzSp, in line with a previous study documenting the specificity of SDs in such a diagnostically heterogeneous sample [20]. Facial mimicry (electrophysiologically measured by means of facial electromyographic activity, EMG) has been proved to be a mirror motor resonance proxy [31, 41, 42]. For the purpose of this study, we employed a novel electrophysiological paradigm that was specifically designed to evoke congruent facial mimicry through multimodal (i.e. audio and/or video) emotional stimuli. This paradigm was previously demonstrated to be able to detect specific deficits in facial mimicry in schizophrenia patients [25]. 
Table 1. Demographic variables and psychopathological characteristics of the SzSp sample

\begin{tabular}{lrrr}
\hline Characteristic & Mean & SE & $\begin{array}{l}\text { Range } \\
\text { (scale range) }\end{array}$ \\
\hline Age, years & 34.17 & 1.63 & $25-49$ \\
SAPS & 23.12 & 3.83 & $1-58(0-170)$ \\
SANS & 47.41 & 4.12 & $15-83(0-125)$ \\
BSABS & 41.72 & 4.01 & $29-86(0-103)$ \\
Length of illness, years & 11.19 & 1.17 & $2-24$ \\
$\begin{array}{l}\text { Age at first recognized } \\
\quad \text { psychotic episode }\end{array}$ & 24.06 & 1.04 & $19-34$ \\
$\begin{array}{l}\text { Number of hospitalizations } \\
\text { Dose of typical and atypical }\end{array}$ & 3.38 & 0.44 & $0-7$ \\
$\quad$ antipsychotics & & & \\
$\quad$ Dose of atypical antipsychotics & 19.56 & 3.77 & \\
$\quad$ Dose of typical antipsychotics & 6.75 & 1.35 & \\
\hline
\end{tabular}

Drugs are expressed as the cumulative value measured in doseyears in the form of (chlorpromazine equivalent in $\mathrm{mg}) \times($ time on dose measured in years) [46]. SAPS $=$ Scale for the Assessment of Positive Symptoms; SANS = Scale for the Assessment of Negative Symptoms.

\section{Methods}

\section{Sample}

The sample included 18 outpatients ( 13 males, 5 females, mean age \pm SE: $34.17 \pm 1.63$ years) and was recruited at the Psychiatry Division of Parma University, Department of Neuroscience. All participants were diagnosed with a SzSp disorder (i.e. schizophrenia or schizotypal personality disorder according to DSM-IV diagnostic criteria) [43] and were clinically stable (i.e. with no current psychotic symptoms) at the time of the experimental assessment. Data from 14 participants included in this group were previously reported in a separate validation study [25] using the same paradigm. This study focused on different questions than the one currently at stake, namely the comparison of behavioral and EMG data detected from schizophrenia patients with respect to healthy participants. The results reported in the current study are therefore entirely original, while considering clinical data acquired from the BSABS interview.

Before inclusion, all patients were screened to rule out any history of neurological and vascular disorders, alcohol or drugs abuse and mental retardation. Clinical features were documented with the Scales for the Assessment of Positive and Negative Symptoms $[44,45]$, whereas disturbances of subjective experience were explored through the BSABS $[2,11]$ (see the online suppl. material section for details; for all online suppl. material, see www.karger. com/doi/10.1159/000380884). Since all patients were under psychopharmacologic treatment with antipsychotics, cumulative measure of lifetime drug exposure was calculated following Andreasen et al. [46]. Demographic and clinical features of the sample are provided in table 1 .

Written informed consent was obtained from all participants before entering the study, after the treating clinician gave them an exhaustive explanation of the study. The Ethics Committee of the University of Parma approved the study that was carried out according to the ethical standards of the 2013 Declaration of Helsinki.

\section{Experimental Paradigm: Stimuli and Procedure}

The experimental paradigm consisted of the following procedure (see [25] for details and empirical validation): participants were presented with 2 -second colored video clips portraying positive (Laugh), negative (Cry) and neutral (Control) emotional stimuli in visual (i.e. Video) and/or auditory (i.e. Audio) modalities. Video or Audio modalities were either in isolation (i.e. Video or Audio alone) or combined (i.e. Audio-Visual). The Audio-Visual combination was either congruent (Audio-Visual Congruent, AVC), i.e. Audio and Video conveying the same emotion (e.g. Cry) or incongruent (Audio-Visual Incongruent, AVI), i.e. Audio and Video conveying contradictory information (for example, in AVI Cry participants saw an actor laughing but heard crying whereas in AVI Laugh participants saw an actor crying but heard laughing). Participants were requested to recognize and quantitatively rate the emotional value of the perceived stimuli, while EMG activity of corrugator supercilii and zygomaticus major muscles (whose activity is evoked by the perception of negative and positive emotions, respectively) was recorded. For further details about stimuli, experimental procedure and EMG recording, see the online supplementary material section and Sestito et al. [25].

\section{Data Analysis}

EMG activity data and behavioral rating scores were analyzed following the same procedure defined in the validation study [25]. The BSABS interviews were conducted by a senior psychiatrist (C.M.) with extensive research interview experience and principal translator of BSABS into Italian, together with experienced psychiatrists (E.L., M.T., R.F., G.D.P.) specifically trained in the use of BSABS. A total amount of 103 items were assessed for presence/ absence (98 principal items plus 5 items exploring coping strategies). Patients were inquired about the anomalies of experience present since illness onset. The average period elapsed between BSABS and EMG assessments was $82.50 \pm$ (SE) 20.96 days. In order to explore possible relations between electrophysiological evidence and phenomenological experiences thought to constitute a distinctive phenotype of schizophrenia psychopathology, EASEanalog subscales were constructed from the available BSABS data. BSABS items were indeed grouped into seven rational scales [2, 47] representing essential dimensions of the SzSp experiential pathology: Diminished Affectivity, Disturbed Contact, Perplexity, Cognitive Disorder, Self-Disorder, Cenesthesias and Perceptual Disorder. To ensure good internal consistency, each scale was subjected to an item analysis, intended to maximize a coefficient [48]. For further details about behavioral and EMG data analyses, see the online supplementary material section.

\section{Results}

\section{EMG Data}

The specific aim of EMG analyses was to detect significant EMG activations with respect to the control condition, in different emotions (Laugh and Cry, that is, 
positive and negative emotions domain), modalities (AVC, AVI, Audio and Video) and time epochs (T1: 0-500 ms; T2: 500-1,000 ms; T3: $1,000-1,500 \mathrm{~ms}$; T4: $1,500-2,000 \mathrm{~ms}$ ), and consider them in the subsequent correlation analysis with BSABS subscores. Indeed, the inclusion in the current paradigm of Control stimuli i.e. effectively judged as emotionally neutral by both patients and healthy participants (see the online suppl. material section and the previous validation study [25]) - allowed us to consider them in post hoc comparisons in order to detect significant EMG activations in the experimental conditions.

EMG activations in SzSp participants emerged in the corrugator muscle for negative emotions (i.e. Cry) during the following conditions: AVC Cry, only at T3 (i.e. the time epoch spanning from 1,000 to $1,500 \mathrm{~ms}$ after stimulus onset); in Audio Cry at T1 and T2 (i.e. from 0 to $1,000 \mathrm{~ms}$ after stimulus onset); in Video Cry at T2, T3, T4 (i.e. from 500 to 2,000 ms after stimulus onset); finally, no activations were found in AVI Laugh.

Furthermore, the analysis performed on the zygomaticus major muscle EMG responses to positive emotions (i.e. Laugh), on the other hand, yielded no significant results. For additional details regarding EMG data analyses, see the online supplementary material section.

\section{Correlations between EMG Activations and BSABS a priori Scales}

After item analyses, five out of the original seven a priori scales were retained for the subsequent correlation analysis, reaching a satisfactory internal consistency ( $a$ value $\geq 0.55$ ): Diminished Affectivity, Cognitive Disorder, Self-Disorder, Cenestesias and Perceptual Disorder (see Appendix for item composition and a coefficients). Prior to formal analysis, we examined correlations between age and sex and the dependent variables (BSABS a priori scales: Diminished Affectivity, Cognitive Disorder, Self-Disorder, Cenesthesias, Perceptual Disorder). Pearson's correlation analysis showed a significant correlation between Perceptual Disorder and age $\left(\mathrm{r}_{18}=0.481\right.$, $\mathrm{p}<0.05$, two-tailed) whereas no significant correlations emerged between sex and the dependent variables.

A multiple regression analysis was conducted in order to determine the variance explained in the five dependent variables (BSABS a priori scales: Diminished Affectivity, Cognitive Disorder, Self-Disorder, Cenesthesias, Perceptual Disorder) including the EMG activations (AVC Cry T3; Audio Cry T1 and T2; Video Cry T2, T3, T4) as predictors. Moreover, semipartial correlations were calculated for variables supposed to be dependent on each other-
Table 2. Pearson's correlations between EMG activations and SelfDisorder subjective experience domain as measured by a priori scales of Parnas et al. [2]

\begin{tabular}{llll}
\hline Self-Disorder & $\mathrm{R}$ & $\mathrm{R}^{2}$ & $\mathrm{p}$ \\
\hline AVC Cry (T3) & 0.024 & 0.001 & 0.924 \\
Audio Cry (T1) & 0.648 & 0.420 & $0.004^{* *}$ \\
Audio Cry (T2) & 0.579 & 0.335 & $0.012^{*}$ \\
Video Cry (T2) & -0.151 & 0.023 & 0.551 \\
Video Cry (T3) & -0.028 & 0.001 & 0.911 \\
Video Cry (T4) & -0.154 & 0.024 & 0.542 \\
\hline
\end{tabular}

${ }^{*} \mathrm{p}<0.05 ;{ }^{* *} \mathrm{p}<0.01$. T1 = Time epoch 0-500 ms; T2 = time epoch 500-1,000 ms; T3 = time epoch 1,000-1,500 ms; T4 = time epoch $1,500-2,000 \mathrm{~ms}$.

that is, EMG activations occurring during consecutive time epochs T1, T2, T3 and T4. This procedure allowed an estimation of the independent contribution of each EMG activation recorded in a given time epoch above and beyond the variance accounted for by the other ones.

Results showed that only the Self-Disorder subscore reached significance $\left(\mathrm{F}_{6,11}=5.83, \mathrm{p}<0.01, \mathrm{R}=0.872, \mathrm{R}^{2}=\right.$ 0.761 ), for which the overall regression model accounted for $76.1 \%$ of the variance. The significant predictor variables for SD were the EMG corrugator supercilii muscle activations detected in the Audio Cry condition, both in $\mathrm{T} 1(\mathrm{t}=3.41, \beta=0.65, \mathrm{p}<0.01)$ and $\mathrm{T} 2(\mathrm{t}=2.84, \beta=0.58$, $\mathrm{p}<0.05$; table 2).

Semipartial correlation coefficients for conditions in which EMG activations occurred during consecutive time epochs (i.e. Audio and Video Cry conditions) were calculated. Only the correlation between SD and the EMG activation of the corrugator muscle occurring during the first time epoch (T1) in the Audio Cry condition was significant $(\mathrm{t}=2.92, \beta=1.89, \mathrm{p}<0.05)$ whereas a negative correlation emerged between SD and the EMG corrugator activation recorded during the Video Cry condition in $\mathrm{T} 4(\mathrm{t}=-2.26, \beta=-0.88, \mathrm{p}<0.05$; table 3$)$.

\section{Discussion}

The study confirms that subtle changes in emotional motor resonance can be detected in SzSp patients - specifically, an important change in facial emotional resonance occurs when participants perceive negative emotional stimuli. Such an imbalance in emotional motor resonance (with a proneness to resonate with negative 
Table 3. Semipartial correlations between EMG activations and Self-Disorder subscale

\begin{tabular}{lrll}
\hline Self-Disorder & \multicolumn{1}{l}{$\mathrm{R}$} & $\mathrm{R}^{2}$ & $\mathrm{p}$ \\
\hline Audio Cry (T1) & 0.430 & 0.185 & $0.014^{*}$ \\
Audio Cry (T2) & -0.257 & 0.066 & 0.110 \\
Video Cry (T2) & 0.085 & 0.007 & 0.578 \\
Video Cry (T3) & 0.067 & 0.005 & 0.658 \\
Video Cry (T4) & -0.333 & 0.111 & $0.045^{*}$ \\
\hline
\end{tabular}

${ }^{*} \mathrm{p}<0.05 . \mathrm{T} 1=$ Time epoch $0-500 \mathrm{~ms} ; \mathrm{T} 2=$ time epoch 500 $1,000 \mathrm{~ms} ; \mathrm{T} 3$ = time epoch 1,000-1,500 ms; T4 = time epoch 1,500$2,000 \mathrm{~ms}$.

stimuli along with a missing resonance to positive stimuli) is consistent with the previous literature [25, 49-54] and might result - on a clinical level - in an aberrant assignment of salience [55] to negative stimuli.

Moreover, some of the EMG proxies of such an altered resonance mechanism (i.e. T1 and T2 EMG corrugator facial activations) strongly predict the occurrence of SDs, selectively when the negative stimuli are presented in auditory modality. A further corroboration of such a robust effect emerged when variance accounted for by other variables was controlled, disclosing the earliest EMG corrugator activation detected during the auditory-only modality to be the specific predictor of SDs, and the latest EMG response occurring during the visual-only modality to predict a reversed effect.

Besides being suggestive of a prominent role of the auditory modality for the perceptual salience of negative stimuli in SzSp, this association may indicate that SzSp-specific qualitative changes in subjectivity (at the phenomenological level) are related to subtle alterations of embodied motor resonance (at the electrophysiological level). That is, alterations affecting the low-level mechanism of facial mimicry (i.e. a basic motor resonance mechanism that normally occurs through the lived body when someone perceives the facial expression of someone else's emotion) are associated with the degree of gestalt/qualitative change in the structure of subjectivity experienced by SzSp patients.

Such an association is in line with the phenomenological conceptualizations of the lived body as the medium of subjectivity/consciousness, in the sense that the impairment of a low-level embodied mechanism (facial mimic resonance) is paralleled by characteristic changes in the structure of conscious experience (i.e. SDs). Indeed - on a phenomenological-experiential level - it is through the organization of the perceptual field that the individual is connected in a 'prereflective' way to the world, precisely through the tacit background of selfawareness that provides the first, elementary basis for his action and cognition $[56,57]$.

Thus, the results of this study are in line with converging evidence suggesting that the disruption of automatic, perceptual processes may be crucially related to a fragmented experience of the self in SzSp patients (see [58] for a comprehensive overview).

The hypothesis of a disturbed intermodal integration was originally proposed by Parnas et al. [59] as a neurodevelopmental feature that may be relevant to the SDs emerging in individuals who develop schizophrenia. Recently, this hypothesis has been corroborated by a prospective high-risk study [60], supporting the importance of intermodality dysfunction as an early indicator of the vulnerability to schizophrenia. On the other hand, an empirical study [61] discussing the view of schizophrenia as a disease mainly characterized by disturbances in information processing emphasized that an imbalance in sensory stimuli perception might arise from a failure in datadriven bottom-up (specifically in our study, facial or bodily feedback) and predominantly concept-driven top-down (e.g. delusional thinking) loop processes. The disruption in the automatic, low-level ability to appropriately integrate sensory inputs or information with stored material indeed can disrupt the sense of continuity and consistency in the sense of the self. Along a similar conceptual line, Postmes et al. [62] recently argued that sensory processing impairments affecting multisensory integration (i.e. multisensory disintegration) and leading to 'perceptual incoherence' might be implicated in the experiential emergence of SDs. These authors hypothesized that an extreme imbalance in different types of sensory input could hinder their integration within a single unified percept, leading to the well-documented multisensory audiovisual disintegration in schizophrenia [63-66]. Indeed, every single sensory modality with its properties contributes to inform the self about the environment via bottomup processes, and conflicting sensory inputs result in sensory ambivalence, bringing forth contradictory experiences. As a consequence, sensory amplification (i.e. an increased impact or salience) of any thought or sensory detail occurs on the one hand, and reduced attention to other events takes place on the other, driving patients to a disintegration of their experiential world. This perceptual incoherence might finally result in a misalignment between mind and bodily self, inducing disturbances of subjective experience such as depersonalization, blurred boundaries, cenesthopathies or diminished sense of own- 
ership and agency. Thus, any deficits in somatosensory feedback (e.g. a lack of facial motor resonance in response to positive emotional stimuli) plus a selective bias toward the 'single-sensory' modalities as found in our study would function as a 'sensory vacuum' that may undermine the perceived bodily self $[40,67]$. We are thus inclined to consider the SzSp alterations in facial motor resonance to Audio-Video emotional stimuli as a proxy of such liability for multisensory disintegration (in the current paradigm: impairment in Audio-Video integration).

Certainly, an interpretation of our results along these lines is somewhat preliminary and has several shortcomings that warrant further investigations in future studies. First, the hypothesis of a disturbed emotional motor resonance derived from abnormalities in the mirror mechanism is limited by the lack in our study of direct measures of the entailed neural mechanisms. Second, all patients had chronic disease and were under antipsychotic medications, which might act as confounders in EMG responses. However, most of them were on second-generation atypical antipsychotics (with reduced extrapyramidal side effects) and additionally, the lack of EMG response was emotion- and modality-specific and not casually distributed among conditions, suggesting a minimal influence of such potential confounders on our data. Also, all patients were clinically stable (i.e. with no frank psychotic symptoms) at the time of the experimental session. Third, the small sample size along with an imbalanced gender distribution might have reduced the statistical power and extension of such results to the general population. Finally, in this study BSABS-derived subscores were adopted instead of the ad hoc developed EASE [12] to map SzSp disorders of self-awareness.

In conclusion, despite its intrinsic limitations, the present study provides the first evidence of a positive correlation between SzSp experiential disorders of the self and a specific neurophysiological substrate (in this case a proxy for the mirror mechanism, that is, EMG facial reactions). Indeed, several lines of investigation have reported empathic response deficits in schizophrenia with abnormalities in the mirror neurons mechanism [31-33]. According to this model, involuntary facial mimicry would act as a relevant low-level mechanism contributing to the experience of empathy via processes of simulation and perception-action coupling subserved by a distributed brain network of interconnected systems (see [68] for a review). Besides, this study confirms the potential of SDs as a target phenotype for neurobiological research and encourages research into disturbed motor/emotional resonance as possible body-level correlate of disturbed sub- jective experiences in $\mathrm{SzSp}$. This may provide a key integrative construct to address future trans-domain research in the neurobiology of vulnerability to schizophrenia.

Furthermore, the paradigm adopted in the current study plays an important heuristic role in mapping the neurodevelopmental processes implicated in SzSp proneness.

\section{Acknowledgments}

This work was supported by the EU grant Towards an Embodied Science of InterSubjectivity (TESIS, FP7-PEOPLE-2010-ITN, 264828) and by a grant from Chiesi Foundation to V.G.

\section{Disclosure Statement}

All the authors have declared that there are no conflicts of interests in relation to the subject of this study.

M.S., A.R. and V.G. jointly developed the heuristic structure of the paper. M.S. wrote the first draft, supervised by A.R. All the authors contributed to the final revision of the manuscript.

\section{Appendix 1}

A priori scales of Parnas et al. [2] with Cronbach's $\alpha$ coefficients and their BSABS item composition:

Diminished Affectivity (DA; $\alpha=0.55)$

Diminished initiative and dynamism (A 4)

Anhedonia (A 6.1)

Diminished feelings for others (A 6.3)

Diminished need for interpersonal relations (A 6.4)

Cognitive Disorder (CD; $\alpha=0.59)$

Though blockages (C 1.4)

Disorder of expressive language (C 1.7)

Diminished thought initiative and goal-directedness of thinking (C 1.13)

Self Disorder (SD; $\alpha=0.73$ )

Psychic depersonalization (B 3.4)

Somatic depersonalization (D 1.1)

Other optic perception disturbances, including the 'mirror phenomenon' (e.g. impression of a change in one's mirror image) (C 2.3)

Cenesthesias (CEN; $\alpha=0.71)$

Electrical bodily sensations (D 5)

Sensation of movement, pressure or pulling in the body or on the body surface (D 7)

Sensation of lightness, heaviness, levitation, falling (D 8)

Sensation of constriction, dilatation, shrinking or expansion of the body (D 9)

Perceptual Disorder (PD; $\alpha=0.62)$

Unclear sight, transitory blindness, partial sight (C 2.1)

Photopsia (C 2.2)

Other optic perception disturbances (C 2.3)

Changes of intensity or quality of acoustic perception (C 2.5)
Sestito et al. 


\section{References}

1 Wieneke A, Schultze-Lutter F, Steinmeyer EM, Klosterkötter J: Abnormal subjective experiences and neuropsychological deficits in first-degree relatives of schizophrenics. Schizophr Res 1997;24:49.

2 Parnas J, Handest P, Saebye D, Jansson L: Anomalies of subjective experience in schizophrenia and psychotic bipolar illness. Acta Psychiatr Scand 2003;108:126-133.

3 Maggini C, Raballo A: Subjective experience of schizotropic vulnerability in siblings of schizophrenics. Psychopathology 2004;37: 23-28.

4 Handest P, Parnas J: Clinical characteristics of first-admitted patients with ICD-10 schizotypal disorder. Br J Psychiatry 2005;187:s49-s54.

5 Raballo A, Parnas J: The silent side of the spectrum: schizotypy and the schizotaxic self. Schizophr Bull 2011;37:1017-1026.

6 Raballo A, Parnas J: Examination of anomalous self-experience: initial study of the structure of self-disorders in schizophrenia spectrum. J Nerv Ment Dis 2012;200:577-583.

7 Raballo A, Sæbye D, Parnas J: Looking at the schizophrenia spectrum through the prism of self-disorders: an empirical study. Schizophr Bull 2011;37:344-351.

8 Parnas J, Handest P: Phenomenology of anomalous self-experience in early schizophrenia. Compr Psychiatry 2003;44:121-134.

9 Parnas J: The self and intentionality in the pre-psychotic stages of schizophrenia; in $\mathrm{Za}$ havi D (ed): Exploring the Self: Philosophical and Psychopathological Perspectives on SelfExperience. Philadelphia, Benjamins, 2000, pp 115-147.

10 Sass LA, Parnas J: Schizophrenia, consciousness, and the self. Schizophr Bull 2003;29: 427-444.

11 Gross G, Huber G, Klosterkötter J, Linz M: Scala di Bonn per la valutazione dei sintomi di base. Edizione italiana a cura di Maggini C e Dalle Luche R. Pisa, ETS Editrice, 1992

12 Parnas J, Møller P, Kircher T, Thalbitzer J, Jansson L, Handest P, Zahavi D: EASE: examination of anomalous self-experience. Psychopathology 2005;38:236-258.

13 Schultze-Lutter F, Addington J, Ruhrmann S, Klosterkötter J: Schizophrenia Proneness Instrument, Adult Version (SPI-A). Rome, Fioriti, 2007.

14 Parnas J, Jansson L, Sass L, Handest P: Selfexperience in the prodromal phases of schizophrenia: a pilot study of first-admissions Neurol Psychiatry Brain Res 1998;6:97-106.

15 Møller P, Husby R: The initial prodrome in schizophrenia: searching for naturalistic core dimensions of experience and behavior. Schizophr Bull 2000;26:217.

16 Klosterkötter J, Hellmich M, Steinmeyer EM, Schultze-Lutter F: Diagnosing schizophrenia in the initial prodromal phase. Arch Gen Psychiatry 2001;58:158-164.

17 Corcoran C, Davidson L, Sills-Shahar R, Nickou C, Malaspina D, Miller T, McGlashan
T: A qualitative research study of the evolution of symptoms in individuals identified as prodromal to psychosis. Psychiatr Q 2003;74: 313-332.

18 Schultze-Lutter F, Ruhrmann S, Berning J, Maier W, Klosterkötter J: Basic symptoms and ultrahigh risk criteria: symptom development in the initial prodromal state. Schizophr Bull 2010;36:182-191.

19 Schultze-Lutter F, Klosterkötter J, Picker H, Steinmeyer E-M, Ruhrmann S: Predicting first-episode psychosis by basic symptom criteria. Clin Neuropsychiatry 2007;4:11-22.

20 Nordgaard J, Parnas J: Self-disorders and the schizophrenia spectrum: a study of 100 first hospital admissions. Schizophr Bull 2014;40: 1300-1307.

21 Nelson B, Thompson A, Yung AR: Basic selfdisturbance predicts psychosis onset in the ultra high risk for psychosis 'prodromal' population. Schizophr Bull 2012;38:1277-1287.

22 Parnas J, Henriksen MG: Disordered self in the schizophrenia spectrum: a clinical and research perspective. Harv Rev Psychiatry 2014; 22:251-265.

23 Nelson B, Whitford T, Lavoie S, Sass L: What are the neurocognitive correlates of basic selfdisturbance in schizophrenia?: integrating phenomenology and neurocognition. Part 1 (Source monitoring deficits). Schizophr Res 2014;152:12-19.

24 Nelson B, Whitford T, Lavoie S, Sass L: What are the neurocognitive correlates of basic selfdisturbance in schizophrenia?: integrating phenomenology and neurocognition. Part 2 (Aberrant salience). Schizophr Res 2014;152: 20-27.

25 Sestito M, Umilta MA, De Paola G, Fortunati R, Raballo A, Leuci E, Maffei S, Tonna M, Amore M, Maggini C, Gallese V: Facial reactions in response to dynamic emotional stimuli in different modalities in patients suffering from schizophrenia: a behavioral and EMG study. Front Hum Neurosci 2013;7:368.

26 Andreasen NC, Calage CA, O'Leary DS: Theory of mind and schizophrenia: a positron emission tomography study of medicationfree patients. Schizophr Bull 2008;34:708719.

27 Derntl B, Finkelmeyer A, Voss B, Eickhoff SB, Kellermann T, Schneider F, Habel U: Neural correlates of the core facets of empathy in schizophrenia. Schizophr Res 2012;136:7081.

28 Kato Y, Mimura M: Disturbance of social interaction in patients with psychosis - a review of the role of malfunction of the mirror neuron system. J Oral Biosci 2012;54:11-14.

29 McCormick LM, Brumm MC, Beadle JN, Paradiso S, Yamada T, Andreasen N: Mirror neuron function, psychosis, and empathy in schizophrenia. Psychiatry Res 2012;201:233-239.

30 Mehta UM, Basavaraju R, Thirthalli J, Gangadhar BN: Mirror neuron dysfunction a neuro-marker for social cognition deficits in drug naïve schizophrenia. Schizophr Res 2012;141:281-283.

31 Derntl B, Finkelmeyer A, Toygar TK, Hülsmann A, Schneider F, Falkenberg DI, Habel U: Generalized deficit in all core components of empathy in schizophrenia. Schizophr Res 2009;108:197-206.

32 Varcin KJ, Bailey PE, Henry JD: Empathic deficits in schizophrenia: the potential role of rapid facial mimicry. J Int Neuropsychol Soc 2010;16:621-629.

33 Gallese V: The roots of empathy: the shared manifold hypothesis and the neural basis of intersubjectivity. Psychopathology 2003;36: 171-180.

34 Gallese V: Embodied simulation: from neurons to phenomenal experience. Phenomenol Cogn Sci 2005;4:23-48.

35 Niedenthal PM: Embodying emotion. Science 2007;316:1002-1005.

36 Niedenthal PM, Winkielman P, Mondillon L, Vermeulen N: Embodiment of emotion concepts. J Pers Soc Psychol 2009;96:1120.

37 Gallese V, Sinigaglia C: The bodily self as power for action. Neuropsychologia 2010;48: 746-755.

38 Gallese V, Sinigaglia C: What is so special about embodied simulation? Trends Cogn Sci 2011;15:512-519.

39 Fuchs T: Corporealized and disembodied minds: a phenomenological view of the body in melancholia and schizophrenia. Philos Psychiat Psychol 2005;12:95-107.

40 Gallese V, Ferri F: Jaspers, the body, and schizophrenia: the bodily self. Psychopathology 2013;46:330-336.

41 Carr L, Iacoboni M, Dubeau M-C, Mazziotta JC, Lenzi GL: Neural mechanisms of empathy in humans: a relay from neural systems for imitation to limbic areas. Proc Natl Acad Sci U S A 2003;100:5497-5502.

42 Seitz R, Schäfer R, Scherfeld D, Friederichs S, Popp K, Wittsack H-J, Azari N, Franz M: Valuating other people's emotional face expression: a combined functional magnetic resonance imaging and electroencephalography study. Neuroscience 2008;152:713-722.

43 American Psychiatric Association: Diagnostic and Statistical Manual of Mental Disorders, ed 4. Washington, American Psychiatric Association, 1994.

44 Andreasen N: Scale for the Assessment of Positive Symptoms (SAPS). Iowa City, University of Iowa, 1984

45 Andreasen NC: Scale for the Assessment of Negative Symptoms (SANS). Iowa City, University of Iowa, 1984.

46 Andreasen NC, Pressler M, Nopoulos P, Miller D, Ho B-C: Antipsychotic dose equivalents and dose-years: a standardized method for comparing exposure to different drugs. Biol Psychiatry 2010;67:255-262.

47 Raballo A, Maggini C: Experiential anomalies and self-centrality in schizophrenia. Psychopathology 2005;38:124-132. 
48 Cronbach LJ: Coefficient alpha and the internal structure of tests. Psychometrica 1951;16: 297-334.

49 Mattes R, Schneider F, Heimann H, Birbaumer N: Reduced emotional response of schizophrenic patients in remission during social interaction. Schizophr Res 1995;17: 249-255.

50 Sison CE, Alpert M, Fudge R, Stern RM: Constricted expressiveness and psychophysiological reactivity in schizophrenia. J Nerv Ment Dis 1996;184:589-597.

51 Iwase M, Yamashita K, Takahashi K, Kajimoto O, Shimizu A, Nishikawa T, Shinosaki K, Sugita Y, Takeda M: Diminished facial expression despite the existence of pleasant emotional experience in schizophrenia. Methods Find Exp Clin Pharmacol 1999;21:189.

52 Kring AM, Earnst KS: Nonverbal behavior in schizophrenia; in Philippot P, Coats E, Feldman RS (eds): Nonverbal Behavior in Clinical Settings. New York, Oxford University Press, 2003, pp 263-286.

53 Wolf K, Mass R, Kiefer F, Wiedemann K, Naber $\mathrm{D}$ : Characterization of the facial expression of emotions in schizophrenia patients: preliminary findings with a new electromyography method. Can J Psychiatry 2006;51:335.

54 Trémeau F: A review of emotion deficits in schizophrenia. Dialogues Clin Neurosci 2006; 8:59-70.
55 Kapur S: Psychosis as a state of aberrant salience: a framework linking biology, phenomenology, and pharmacology in schizophrenia. Am J Psychiatry 2003;160:13-23.

56 Merleau-Ponty M: Phenomenology of Perception, trans. C Smith. London, Routledge, 1962.

57 Merleau-Ponty M: The Primacy of Perception: And Other Essays on Phenomenological Psychology, the Philosophy of Art, History and Politics. Evanston, Northwestern University Press, 1964.

58 Uhlhaas PJ, Mishara AL: Perceptual anomalies in schizophrenia: integrating phenomenology and cognitive neuroscience. Schizophr Bull 2007;33:142-156.

59 Parnas J, Bovet P, Innocenti GM: Schizophrenic trait features, binding, and corticocortical connectivity: a neurodevelopmental pathogenetic hypothesis. Neurol Psychiatry Brain Res 1996;4:185-196.

60 Gamma F, Goldstein JM, Seidman LJ, Fitzmaurice GM, Tsuang MT, Buka SL: Early intermodal integration in offspring of parents with psychosis. Schizophr Bull 2014;40:9921000.

61 Schultze-Lutter F, Ruhrmann S, Hoyer C, Klosterkötter J, Leweke FM: The initial prodrome of schizophrenia: different duration, different underlying deficits? Compr Psychiatry 2007;48:479-488.

62 Postmes L, Sno H, Goedhart S, van der Stel J, Heering H, de Haan L: Schizophrenia as a selfdisorder due to perceptual incoherence. Schizophr Res 2014;152:41-50.
63 de Gelder B, Vroomen J, de Jong SJ, Masthoff ED, Trompenaars FJ, Hodiamont P: Multisensory integration of emotional faces and voices in schizophrenics. Schizophr Res 2005; 72:195-203.

64 De Jong J, Hodiamont P, De Gelder B: Modality-specific attention and multisensory integration of emotions in schizophrenia: reduced regulatory effects. Schizophr Res 2010 122:136-143.

65 De Jong J, Hodiamont P, Van den Stock J, De Gelder B: Audiovisual emotion recognition in schizophrenia: reduced integration of facial and vocal affect. Schizophr Res 2009;107:286293.

66 Castagna F, Montemagni C, Maria Milani A, Rocca G, Rocca P, Casacchia M, Bogetto F: Prosody recognition and audiovisual emotion matching in schizophrenia: the contribution of cognition and psychopathology. Psychiatry Res 2013;205:192-198.

67 Ferri F, Frassinetti F, Mastrangelo F, Salone A, Ferro FM, Gallese V: Bodily self and schizophrenia: the loss of implicit self-body knowledge. Conscious Cogn 2012;21:13651374.

68 Mehta UM, Thirthalli J, Aneelraj D, Jadhav P, Gangadhar BN, Keshavan MS: Mirror neuron dysfunction in schizophrenia and its functional implications: a systematic review. Schizophr Res 2014;160:9-19.

\section{(C) Free Author Copy - for per- sonal use only \\ ANY DISTRIBUTION OF THIS \\ ARTICLE WITHOUT WRITTEN \\ CONSENT FROM S. KARGER \\ AG, BASEL ISA VIOLATION OF THE COPYRIGHT. \\ Written permission to distrib- ute the PDF will be granted against payment of a per- mission fee, which is based on the number of accesses required. Please contact permission@karger.com}

